\title{
Prevalência das posições de terceiros molares retidos em radiografias panorâmicas: estudo retrospectivo no sertão nordestino
}

\author{
Prevalence of retained third molars positions in panoramic radiographs: \\ a retrospective study in Brazilian Northeastern Sertão
}

\author{
Prevalencia de las posiciones de terceros molares retenidos en radiografías \\ panorámicas: estudio retrospectivo en el sertão nordestino de Brasil \\ João Paulo de Farias GOMES ${ }^{1}$ \\ Julliana Cariry Palhano FREIRE ${ }^{2}$ \\ Jaqueline Oliveira BARRETO ${ }^{3}$ \\ Jalber Almeida dos SANTOS ${ }^{4}$ \\ José Cadmo Wanderley Peregrino de ARAUJO-FILHO ${ }^{5}$ \\ Eduardo DIAS-RIBEIRO ${ }^{6}$ \\ ${ }^{l}$ Cirurgião-Dentista - Clínica Privada, Patos-PB, Brasil \\ ${ }^{2}$ Mestranda em Ciências Odontológicas, Universidade Federal da Paraíba, João Pessoa-PB, Brasil \\ Graduanda em Odontologia, Universidade Federal de Campina Grande, Patos-PB, Brasil \\ ${ }^{4}$ Professor-Mestre. Departamento de Radiologia Odontológica, \\ Faculdades Integradas de Patos, Patos-PB, Brasil \\ ${ }^{5}$ Professor-Mestre. Departamento de Cirurgia e Traumatologia Bucomaxilofacial, \\ Universidade Federal de Campina Grande, Patos-PB, Brasil \\ ${ }^{6}$ Professor-Doutor. Departamento de Cirurgia e Traumatologia Bucomaxilofacial, \\ Universidade Federal de Campina Grande, Patos-PB, Brasil
}

\begin{abstract}
Resumo
O estudo avaliou o posicionamento dos terceiros molares inclusos segundo as classificações de Winter e Pell e Gregory, bem como avaliar a prevalência por sexo e faixa etária. Foi realizado um estudo documental, retrospectivo, descritivo, através de análises de radiografias ortopantomográficas que fazem parte do banco de dados da Clínica Radiológica de Patos, Patos, Paraíba, Brasil. Foram analisadas 100 radiografias pertencentes a pacientes entre 20 e 40 anos, das quais $63 \%$ pertenciam ao sexo feminino e $44 \%$ correspondia à faixa etária de 20 a 25 anos. Foram observados 270 dentes inclusos, sendo 112 na maxila e 158 na mandíbula. Com relação à classificação de Winter, a posição mais prevalente na maxila foi vertical, já na mandíbula foi mesioangular. Considerando a classificação de Pell e Gregory, quando avalia a profundidade, a posição mais presente foi a posição B, e quanto a borda anterior do ramo da mandíbula a classe II. A prevalência do terceiro molar incluso foi maior no sexo feminino, na faixa etária de 20 a 25 anos, com relação à maxila, a posição que prevaleceu foi a vertical e na mandíbula foi a mesioangular e a posição B e classe II.

Descritores: Classificação; Dente Impactado; Dente Serotino.
\end{abstract}

\begin{abstract}
The study evaluated the positioning of impacted third molars in the classifications of Winter and Pell and Gregory, as well as to evaluate the prevalence by gender and age group. A retrospective, descriptive, documentary study was carried out through analyzes of orthopantomographic radiographs that are part of the database of the Radiological Clinic of Patos, Patos city, Paraíba, Brazil. We analyzed 100 radiographs belonging to patients between 20 and 40 years of age, of which $63 \%$ belonged to the female gender and $44 \%$ corresponded to the age group of 20 to 25 years. 270 impacted teeth were observed, 112 being in the maxilla and 158 in the mandible. Regarding the classification of Winter, the most prevalent in the maxilla was vertical, already in the mandible was mesioangular. Considering the classification of Pell and Gregory, when evaluating the depth the most present position was position $\mathrm{B}$, and when anterior border of the mandible branch, class II. The prevalence of the impacted third molar was higher in the female gender, in the age group of 20 to 25 years, with regard to the maxilla, the position that prevailed was vertical and the mandible was mesioangular and position B and Class II.
\end{abstract}

Descriptors: Classification; Tooth, Impacted; Molar, Third.

\section{Resumen}

El estudio evaluó el posicionamiento de los terceros molares retenidos según las clasificaciones de Winter y Pell y Gregory, así como evaluar la prevalencia por género y grupo de edad. Se realizó un estudio documental, retrospectivo, descriptivo, a través de análisis de radiografías ortopantomográficas que forman parte del banco de datos de la Clínica Radiológica de Patos, Patos, Paraíba, Brasil. Se analizaron 100 radiografías pertenecientes a pacientes entre 20 y 40 años, de las cuales el $63 \%$ pertenecía al género femenino y el $44 \%$ correspondía al grupo de edad de 20 a 25 años. Se observaron 270 dientes retenidos, siendo 112 en la maxila y 158 en la mandíbula. Con respecto a la clasificación de Winter, la posición más prevalente en la maxila fue vertical, ya en la mandíbula fue mesioangular. En cuanto a la clasificación de Pell y Gregory, cuando evalúa la profundidad, la posición más presente fue la posición $\mathrm{B}$, y en cuanto al borde anterior de la rama de la mandíbula a la clase II. La prevalencia del tercer molar retenido fue mayor en el género femenino, en la franja de edad de 20 a 25 años, con relación a la maxila, la posición que prevaleció fue la vertical y en la mandíbula fue la mesiogonal y la posición B y clase II.

Descriptores: Clasificación; Diente Impactado; Tercer Molar

\section{INTRODUÇÃO}

O sistema estomatognático sofreu diversas modificações e evoluiu de acordo com o tipo de alimentação do homem durante vários anos. Em vista disso, alterações foram percebidas nos maxilares, como a extinção do quarto molar, que é considerado hoje como um supranumerário e a incidência cada vez maior de anodontia e inclusão dos terceiros molares, principal dente com indicação de exodontia baseada em análise de radiografias panorâmicas e periapicais para um correto planejamento cirúrgico ${ }^{1-4}$.

Os terceiros molares são os elementos dentários que 
apresentam maior frequência de inclusão, ou seja, chegaram ao tempo normal de erupcionar, mas permaneceram encobertos no interior do tecido ósseo ou submucoso, de forma parcial ou total ${ }^{1,4}$. Sua remoção é preconizada quando estiver associado à patologia ou se o paciente apresentar sintomatologia dolorosa ${ }^{5,6}$. Para isso, a avaliação radiográfica é de suma importância para classificação desses elementos dentários quanto a sua posição, pois fornece informações de sua relação com estruturas anatômicas e possíveis alterações que possam estar associados, permitindo o correto estabelecimento do tipo de tratamento a ser selecionado $^{7,8}$.

Os motivos para a impacção são a angulação incorreta do dente, espaço retromolar insuficiente, e presença de lesões patológicas impedindo mecanicamente o trajeto de erupção ${ }^{8}$. Graziani ${ }^{9}$ relata as prováveis causas da inclusão e má posição do terceiro molar inferior: falta de espaço na arcada em virtude de menor desenvolvimento mandibular; dentes muito volumosos; obstáculo oferecido por dente vizinho; resistência demasiada oferecida pelo tecido ósseo; obstáculo oferecido por densidade ou processo inflamatório da fibromucosa; permanência exagerada dos dentes temporários; perda prematura dos dentes temporários, alterando a posição dos permanentes.

A fim de facilitar o procedimento cirúrgico, George Winter ${ }^{10}$ e Pell e Gregory ${ }^{11}$ fizeram classificações relevantes dos terceiros molares, sendo a classificação do primeiro autor de acordo com a inclinação do seu longo eixo em relação ao do segundo molar, estabelecendo os seguintes grupos: vertical, mesioangular, distoangular, horizontal, vestibuloangular, linguoangular e invertido. Enquanto Pell e Gregory $^{11}$ desenvolveram duas formas distintas de classificação para os terceiros molares inclusos: um que determina a posição do $3^{\circ}$ molar inferior em sua relação com o ramo da mandíbula (classe I, classe II ou classe III) e a segunda, relacionada ao plano oclusal do segundo molar (posição A, posição B ou posição C).

O conhecimento da prevalência dos terceiros molares inclusos é importante, pois serve como indicativo para o cirurgião bucomaxilofacial sobre a estimativa do número de pacientes que tendem a passar por procedimento cirúrgico para remoção dos terceiros molares. Quanto ao posicionamento e à relação com outras estruturas, os resultados podem ser úteis aos cirurgiões para que, baseados nas informações, possam fazer um planejamento cirúrgico mais cuidadoso, a fim de evitar maiores complicações no trans e pós-operatório.

O presente estudo objetivou investigar a prevalência do posicionamento e da situação dos terceiros molares superiores e inferiores no complexo maxilomandibular em pacientes atendidos em uma Faculdade de Odontologia do sertão nordestino, por meio de radiografias panorâmicas, classificando os terceiros molares de acordo com Winter ${ }^{10} \mathrm{e}$ Pell e Gregory ${ }^{11}$.

\section{MATERIAL E MÉTODO}

O presente estudo foi aprovado pelo Comitê de Ética em Pesquisa das Faculdades Integradas de Patos (FIP), Patos, Paraíba, Brazil, com base na Resolução 466/2012 do Conselho Nacional de Saúde do Ministério da Saúde, Brazil, sob número de parecer 991.992 .

Trata-se de uma pesquisa documental retrospectiva, com dados secundários que analisou radiografias ortopantomográficas digitalizadas através do banco de prontuários da Clínica Radiográfica de Patos. De um total de 700 radiografias, 100 foram selecionados, de forma que pertencesse a pacientes na faixa etária de 20 a 40 anos e que não apresentavam ausência de terceiros molares retidos.

As imagens foram analisadas por um único examinador com ajuda de um computador (Intel Core ${ }^{\mathrm{TM}} \mathrm{i}$, Intel ${ }^{\circledR}$ Corp., USA). Para realização dessa coleta foi utilizada uma ficha específica pré-elaborada que registrou o sexo, idade (estratificada a cada 5 anos) e os terceiros molares retidos com suas respectivas classificações de Winter ${ }^{10} \mathrm{e}$ Pell e Gregory ${ }^{11}$ e por fim, para análise das imagens, foi utilizado o programa Microsoft Office Picture Manager (Office para Windows ${ }^{\circledR}$ 7, Califórnia, USA).

A análise dos dados coletados foi realizada por meio de estatística descritiva, utilizando o programa Microsoft Office Excel (Office para Windows ${ }^{\circledR}$ 7, Califórnia, USA), que permitiu elaborar a distribuição de frequência das variáveis consideradas neste estudo por meio de tabelas e gráficos, para avaliar a prevalência de terceiros molares retidos e sua localização.

\section{RESULTADOS}

A partir de uma amostra de 100 radiografias ortopantomográficas, foram observados 270 dentes inclusos, dos quais $171(63 \%)$ pertenciam a pacientes do sexo feminino e 99 (37\%) pertencia ao sexo masculino. A maior prevalência por faixa etária foi observada no grupo de 20 a 25 anos, com índice de (44\%), seguido do grupo de 26 a 30 com (33\%), de 31 a 35 anos (17\%) e de 36 a 40 com apenas (6\%). Na maxila foram observados 112 dentes inclusos. De acordo com a classificação de Winter ${ }^{10}$, a posição mais prevalente foi a vertical (V), com $44 \%$ dos casos, seguida da distoangular (DA) com 34,5\% e mesioangular (MA) com 15\%. Em 6,5\% dos casos, sugeriu-se a posição vestíbuloversão (VA) (Gráfico 1). Não foi encontrado nenhum dente nas posições horizontal e invertida. $\mathrm{Na}$ mandíbula, a presença de dentes inclusos foi bem maior, com 158 dentes inclusos. Destes, a maioria se encontrava na posição mesioangular (37\%), seguido da posição vertical (31\%), horizontal com $25,4 \%$ e apenas $0,6 \%$ sugeriu-se estar na vestíbuloversão (Gráfico 2). Não foi encontrado nenhum dente na posição invertida.

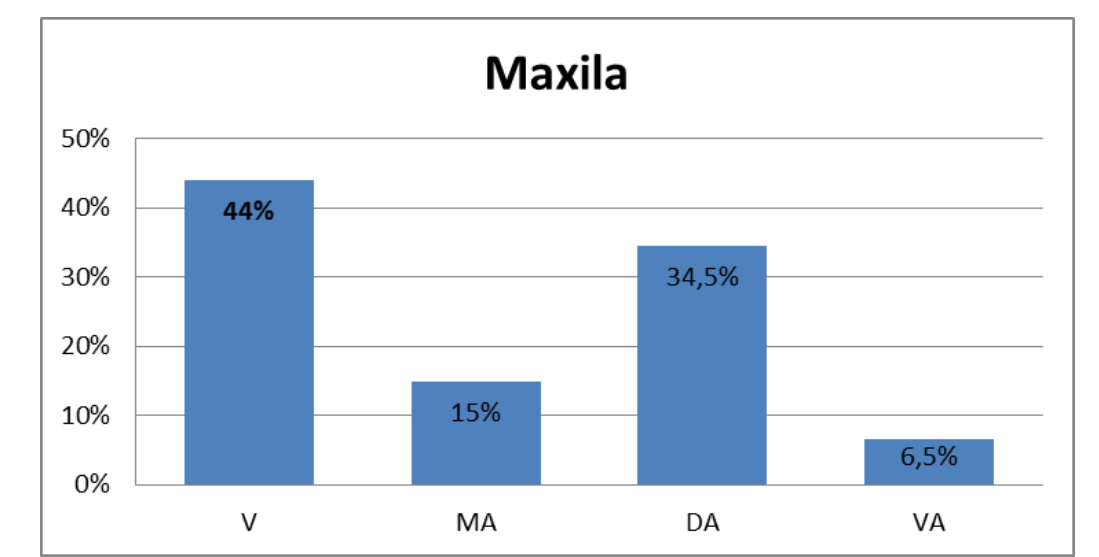

Gráfico 1: Prevalência de terceiro molar incluso na maxila de acordo com a classificação de Winter ${ }^{10}$

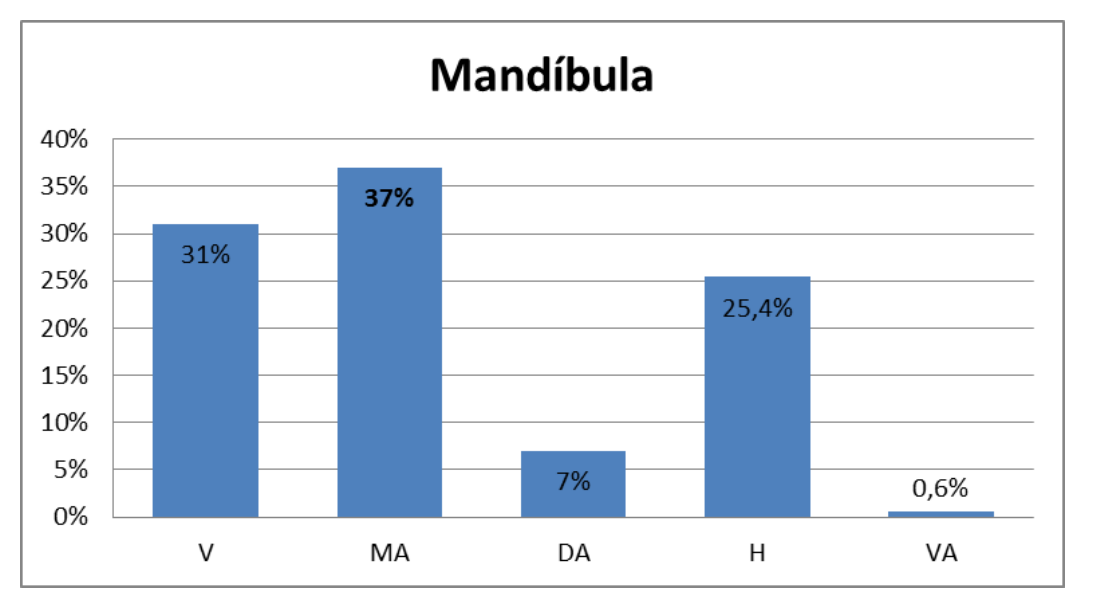

Gráfico 2: Prevalência do terceiro molar incluso na mandíbula de acordo com a classificação de Winter ${ }^{10}$ 
Conforme classificação desenvolvida por Pell e Gregory ${ }^{11}$, quanto à profundidade de inclusão, a posição prevalente na amostra estudada foi posição $\mathrm{B}$, com $66 \%$, acompanhado pela posição A (com $26 \%$ ) e posição C (com $8 \%$ ) como se observa na Tabela 1 . Já em relação ao ramo da mandíbula, a classe II foi a mais prevalente neste estudo, apresentando $52 \%$ dos casos, seguida pela classe I com $45,5 \%$ e classe III com apenas $2,5 \%$ (Tabela 2 ).

Tabela 1. Posições dos terceiros molares inclusos na mandíbula, quanto à profundidade de inclusão de acordo com a classificação Pell e Gregory ${ }^{11}$

\begin{tabular}{ccc}
\hline POSIÇÕES & n & \% \\
\hline A & 41 & $26 \%$ \\
B & 105 & $66 \%$ \\
C & 12 & $8 \%$ \\
Total & 158 & $100 \%$ \\
\hline
\end{tabular}

Tabela 2. Posições dos terceiros molares inclusos na mandíbula, quanto à relação com ramo da mandíbula, de acordo com a classificação Pell e Gregory $^{11}$

\begin{tabular}{ccc}
\hline POSIÇÕES & $\mathbf{n}$ & $\mathbf{\%}$ \\
\hline Classe I & 73 & $45,5 \%$ \\
Classe II & 82 & $52 \%$ \\
Classe III & 03 & $2,5 \%$ \\
Total & 158 & $100 \%$ \\
\hline
\end{tabular}

\section{DISCUSSÃO}

Em relação ao sexo, o maior percentual de prevalência foi o feminino, correspondente a $63 \%$ das radiografias observadas. Resultados semelhantes foram encontrados por Pinto et al. ${ }^{12}$, Cerqueira et al. ${ }^{7}$, Garcia et al. $^{13}$, Hattab et al. ${ }^{14}$, Vasconcellos et al. ${ }^{15}$ e Marzola et al. ${ }^{16}$, sugerindo que o maior percentual de pacientes desse sexo é devido a uma maior preocupação deste grupo com a saúde bucal, motivando uma maior procura pelos serviços de saúde. Todavia, discordam dos resultados encontrados por Hassan $^{17}$ e Trento et al. ${ }^{18}$, os quais observaram que o sexo masculino era o mais prevalente, enquanto Jaffar e Tin-Oo ${ }^{19}$, Vannucci et al. ${ }^{20}$ e Topkara e Sari $^{21}$ não encontraram diferenças estatísticas.

Quanto à faixa etária, no presente estudo verificou que há predominância de terceiros molares inclusos entre os 20 a 25 anos, seguida da faixa etária dos 26 -30 anos. Este resultado está em conformidade com a grande maioria dos estudos que fundamentam este trabalho a exemplo de Marinho et al. ${ }^{22}$, Garcia et al. ${ }^{13}$, Dias-Ribeiro et al. ${ }^{2}$, Pinto et al. ${ }^{12}$. Infere-se para essa alta incidência algumas hipóteses: cronologia de erupção dos terceiros molares serem em torno de 21 anos de idade, logo dentro dessa faixa etária; alterações na estrutura óssea dos maxilares ao longo da história da espécie, provocadas por mudanças nos hábitos alimentares, levando a uma diminuição do perímetro das arcadas dentarias, o que retardaria a erupção, como nota-se a incidência do elemento em análise decair conforme aumenta a idade dos grupos: $44 \%$ no grupo de 20 a 25 anos, $33 \%$ no grupo de 26 a 30 anos, $17 \%$ no grupo de 31 a 35 anos e $6 \%$ no grupo de 36 a 40 anos.

Em comparação com outros estudos, como Al-Anqudi et al. ${ }^{23}$, Farias et al. ${ }^{24}$, Dias-Ribeiro et al. ${ }^{4}$, Marzola et al. ${ }^{16}$, Topkara e Sari ${ }^{21}$, a prevalência de dentes inclusos foi mais observada na mandíbula. Discordando de um único estudo encontrado, o de Xavier et al. ${ }^{25}$, o qual prevaleceu os dentes retidos na maxila.

Segundo a classificação de Winter $^{10}$, para os dentes da maxila foi visto uma predominância na posição vertical e na mandíbula, mesioangular. Resultados estes, que se assemelham com o de Pinto et al. ${ }^{12}$ e Xavier et al. ${ }^{25}$.

Entretanto, discordam com Dias-Ribeiro et al. ${ }^{2}$, Lisboa et al. ${ }^{3}$, Topkara e Sari ${ }^{21}$, Eshghpour et al. ${ }^{26}$ e AlAnqudi et al. $^{23}$, os quais a posição mesioangular na maxila e vertical e distoangular na mandíbula prevaleceu.

Conforme a classificação de Pell e Gregory ${ }^{11}$, a posição prevalente foi a posição $\mathrm{B}$, em relação a profundidade de inclusão e a classe II, considerando a relação com o ramo da mandíbula. Estes resultados são similares aos encontrados por Eshghpour et al. ${ }^{26}$, Lisboa et al. ${ }^{3}$. Este último autor, concluiu que a maioria dos dentes inferiores situava-se entre a borda anterior do ramo da mandíbula e a face distal do segundo molar, tendo o seu plano oclusal entre o colo anatômico e a superfície oclusal do segundo molar.

Entre as diferenças, destacamos a prevalência da classe II e posição B na classificação de Pell e Gregory ${ }^{11}$, enquanto em outros estudos como o de Xavier et al. ${ }^{25}$ e Pinto et al. ${ }^{12}$ mostrou a grande prevalência da posição A e SantosJúnior et al. ${ }^{27}$ mostrou que predomina a classe I e posição A. Esta pequena diferença pode decorrer por questões estatísticas das amostras analisadas nos diferentes estudos.

De modo geral, os resultados encontrados nesta investigação assemelham-se relevantemente aos estudos anteriormente realizados sobre a temática. $\mathrm{O}$ estudo da prevalência e posição dos terceiros molares através de radiografias se mostra de grande importância para o diagnóstico e planejamento da remoção desses elementos, assim como para a prevenção de complicações no transoperatório.

\section{CONCLUSÃO}

Conclui-se que a prevalência do terceiro molar incluso foi maior no sexo feminino, na faixa etária de 20 a 25 anos. A posição que prevaleceu na maxila segundo a classificação de Winter foi a vertical e na mandíbula foi a mesioangular. Já de acordo com a classificação de Pell e Gregory, teve mais prevalência a posição B e classe II.

\section{REFERÊNCIAS}

1. Santos DR, Quesada GAT. Prevalência de terceiros molares e suas respectivas posições segundo as classificações de Winter e de Pell e Gregory. Rev Cir Traumatol Buco-Maxilo-fac. 2009; 9(1):83-92.

2. Dias-Ribeiro E, Lima-Júnior JL, Barbosa JL, Haagsma IB, Lucena LBS. Prevalencia de la posición de terceros molares inferiores retenidos con relación a la clasificación de Pell \& Gregory. Rev Odontol Mex. 2009; 13(4):229-33.

3. Lisboa AH, Gomes G, Hasselman Junior EA, Pilatti GL. Prevalência de inclinações e profundidade de terceiros molares inferiores, segundo as classificações de Winter e de Pell \& Gregory. Pesq Bras Odontoped Clin Integr. 2012; 12(4):511-5.

4. Dias-Ribeiro E, Lima-Júnior JL, Barbosa JL, Haagsma IB, Lucena LBS, Marzola C. Avaliação das posições de terceiros molares retidos em relação à classificação de Winter. Rev Odontol UNESP. 2008; 37(3):203-9.

5. Sant'Ana E, Ferreira-Júnior O, Pinzan CRM. Avaliação da frequência da posição dos terceiros molares inferiores não irrompidos. Rev Bras Cir Implan. 2000; 7(27):42-5. 
6. Oliveira MG, Becker EM, Spohr AM, Zeni E. Radiografia panorâmica na complementação diagnóstica de inclusões de terceiros molares. Rev Odonto Cienc. 1996; 11(22):83-91.

7. Cerqueira PRF, Farias DLB, Silva Filho JP, Oliveira TQF. Análise da topografia axial dos terceiros molares inclusos através da radiografia panorâmica dos maxilares em relação à classificação de Winter. Rev Odonto Cienc. 2007; 22(55):16-22.

8. Różyło-Kalinowska I, Burdan F, Marchut $\mathrm{T}$. Morphology of third molar teeth with incompletely formed apices on the basis of panoramic radiograms. Folia Morphol. 2003; 62(2):113-7.

9. Graziani, M. Cirurgia Bucomaxilofacial. 8th ed., Rio de Janeiro: Guanabara Koogan; 1997.

10. Winter GB. Impacted mandibular third molars. St. Louis: American Medical Book; 1926.

11. Pell GJ, Gregory BT. Impacted mandibular third molars classifications and modified technique for removal. Dent Digest. 1933; 39(9):330-8.

12. Pinto DG, Mockdeci HR, Almeida LE, Assis NMSP, Vilela EM. Análise da prevalência e correlações por gênero, faixa etária, raça e classificação dos terceiros molares. HU Revista. 2015; 41(3/4):155-62.

13. Garcia RR, Paza AO, Moreira RWF, Moraes M, Passeri LA. Avaliação radiográfica da posição de terceiros molares inferiores segundo as classificações de Pell \& Gregory e Winter. RFO/UPF. 2000; 5(2):31-6.

14. Hattab FN. Positional changes and eruption of impacted mandibular third molars in young adults. A radiographic 4-year follow-up study. Oral Surg Oral Med Oral Pathol Oral Radiol Endod. 1997; 84(6):604-8.

15. Vasconcellos RJH, Oliveira DM, Moreira MD, Fulco MHM. Incidência dos terceiros molares retidos em relação à classificação de Winter. Rev Cir Traumatol Buco-Maxilo-fac. 2002; 2(1):43-7.

16. Marzola C, Comparin E, Toledo-Filho JL. Third molars classifications prevalence in the cities of Cunha Porã, Maravilha and Palmitos in the northwest of Santa Catarina state in Brazil. Rev Odonto Cienc. 2006; 21(51):55-66.

17. Hassan AH. Pattern of third molar impaction in a Saudi population. Clin Cosmet Investig Dent. 2010; 2:109-13.

18. Tentro CL, Zini MM, Moreschi E, Zamponi M, Gottardo DV, Cariani JP. Localização e classificação de terceiros molares: análise radiográfica. Interbio. 2009; 3(2):18-26.

19. Jaffar RO, Tin-Oo MM. Impacted mandibular third molars among patients attending Hospital Universiti Sains Malaysia. Arch Orofac Scienc. 2009; 4(1):7-12.

20. Vannucci MG, Fritzen TN, Moraes JFD, Weber JBB, Hellwig I, Oliveira MG et al. Estudo comparativo da variabilidade da posição dos terceiros molares retidos em pacientes adolescentes e adultos jovens. Stomatos. 2010; 16(31):4-13.

21. Topkara A, Sari Z. Investigation of third molar impaction in Turkish orthodontic patients: Prevalence, depth and angular positions. Eur J Dent. 2013; 7(Suppl 1):S94-S98.

22. Marinho SA, Verli FD, Amenabar JM, Brucker MR. Avaliação da posição dos terceiros molares inferiores retidos em radiografias panorâmicas. Robrac: Rev Odontol Brasil Central. 2005; 14(37):65-8.

23. Al-Anqudi SM, Al-Sudairy S, Al-Hosni A, Al-Maniri A Prevalence and pattern of third molar impaction: A retrospective study of radiographs in Oman. Sultan Qaboos Univ Med J. 2014;14(3):e388-92.
24. Farias JG, Santos FAP, Campos PSF, Sarmento VA, Barreto S, Rios V. Prevalência de dentes inclusos em pacientes atendidos na disciplina de cirurgia do curso de odontologia da universidade estadual de Feira de Santana. Pesq Bras Odontoped Clin Integr. 2003; 3(2):15-9.

25. Xavier CRG, Dias-Ribeiro E, Ferreira-Rocha J, Duarte BG, Ferreira-Júnior O, Sant'Ana E et al. Avaliação das posições dos terceiros molares impactados de acordo com as classificações de Winter e Pell \& Gregory em radiografias panorâmicas. Rev Cir Traumatol BucoMaxilo-fac. 2010; 10(2): 83-90.

26. Eshghpour M, Nezadi A, Moradi A, Shamsabadi R M, Rezaei N M, Nejat A. Pattern of mandibular third molar impaction: A cross-sectional study in northeast of Iran. Niger J Clin Pract. 2014; 17(6):673-7

27. Santos-Júnior PV, Marson JO, Toyama RV, Santos JRC. Terceiros molares inclusos mandibulares: incidência de suas inclinações, segundo classificação de Winter: levantamento radiográfico de 700 casos. RGO. 2007; 55(2):143-7.

\section{CONFLITO DE INTERESSES}

Os autores declaram não haver conflitos de interesse.

\section{AUTOR PARA CORRESPONDÊNCIA}

\section{Eduardo Dias Ribeiro}

eduardodonto@yahoo.com.br

Submetido em 19/04/2017 Aceito em 06/06/2017 